\title{
Constructive Pointfree Topology Eliminates Non-constructive Representation Theorems from Riesz Space Theory
}

\section{Bas Spitters}

Received: 8 June 2009 / Accepted: 5 February 2010 / Published online: 10 March 2010

(C) The Author(s) 2010. This article is published with open access at Springerlink.com

\begin{abstract}
In Riesz space theory it is good practice to avoid representation theorems which depend on the axiom of choice. Here we present a general methodology to do this using pointfree topology. To illustrate the technique we show that Archimedean almost f-algebras are commutative. The proof is obtained relatively straightforward from the proof by Buskes and van Rooij by using the pointfree Stone-Yosida representation theorem by Coquand and Spitters.
\end{abstract}

Keywords Formal topology $\cdot$ Axiom of choice $\cdot$ Riesz space $\cdot$ Constructive analysis

The Stone-Yosida representation theorem [25, 28] for Riesz spaces shows how to embed every Riesz space [20,29] into the Riesz space of continuous functions on its spectrum.

Definition 1 A Riesz space is a vector space with compatible lattice operationsi.e. $f \wedge g+f \vee g=f+g$ and if $f \geqslant 0$ and $a \geqslant 0$, then $a f \geqslant 0$. A (strong) unit 1 in a Riesz space is an element such that for all $f$ there exists a natural number $n$ such that $|f| \leqslant n \cdot 1$. A Riesz space is Archimedean if for all $n, n|x| \leqslant y$ implies that $x=0$.

A Riesz space with a strong unit is Archimedean.

Definition 2 A representation of a Riesz space $R$ with unit is a linear map $\sigma: R \rightarrow \mathbb{R}$ which respects the lattice operations and the unit. We denote by $\Sigma$ the space of representations equipped with the weakest topology which makes all the maps $e_{r}(\sigma):=\sigma(r)$ continuous.

B. Spitters $(\bowtie)$

Computer Science, Radboud University of Nijmegen, Nijmegen, The Netherlands

e-mail: spitters@cs.ru.nl 
Theorem 1 [Stone-Yosida] Let $R$ be an Archimedean Riesz space (vector lattice) with unit. Let $\Sigma$ be its (compact Hausdorff) space of representations. Define the continuous function $\hat{r}(\sigma):=\sigma(r)$ on $\Sigma$. Then $r \mapsto \hat{r}$ is a Riesz embedding of $R$ into $C(\Sigma, \mathbb{R})$.

The theorem is very convenient, but sometimes better avoided. To quote Zaanen [30]:

Direct proofs, although sometimes a little longer than proofs by means of representation [theorems], often reveal more about the situation under discussion.

Similar concerns where discussed by Buskes, de Pagter and van Rooij [1, 7]. They proposed to avoid the use of the axiom of choice by restricting the size of the Riesz spaces [7]. We provide a solution based on pointfree topology, which like [7] avoids also the countable axiom of choice, but moreover avoids the axiom of excluded middle. This allows the results to be applied in non-standard contexts. For instance, one can translate a theorem about one $\mathrm{C}^{*}$-algebra to a theorem about a continuous field of $C^{*}$-algebras [3-6]. In turn, the results about commutative $C^{*}$-algebras may be obtained directly using Riesz spaces [14]. This is used in applications of topos theory to quantum theory $[10,16,17]$.

Our strategy is as follows. First we replace the topological space of representations by a locale, a point-free space. This typically removes the need of the axiom of choice [22]. Then we proceed to use pointfree topology, locale theory, using only a basis for the topology [24]. Since the space of representations of a Riesz space is compact Hausdorff, it can be described explicitly by the finite covering relation on the lattice of basic opens. This lattice can be defined directly using the Riesz space structure $[12,13]$.

To illustrate the method we will reprove the results by Buskes and van Rooij [8].

\section{Preliminaries}

\subsection{Topologies, Locales, Lattices}

A topological space may be presented in its familiar set theoretic form. As such it is a complete distributive lattice of open sets with the operations of union and intersection. The category of frames has as objects lattices with a finitary meets and infinitary joins such that $\wedge$ distributes over $\bigvee$. Frame maps preserve this structure. A continuous function $f: X \rightarrow Y$ defines a frame map $f^{-1}: O(Y) \rightarrow O(X)$. Since this map goes in the reverse direction it is often convenient to consider the category of locales, the opposite of the category of frames. In fact, there is a categorical adjunction between the category of locales and the category of topological spaces. This restricts to an equivalence of categories for compact Hausdorff spaces and compact regular locales. In general, the axiom of choice is needed to move from locales to topological spaces. Hence, by staying on the localic side it is often possible to avoid the axiom of choice. However, one can go even further. A compact regular 
locales may be presented by a finitary covering relation on a base of the topology. This base is a normal ${ }^{1}$ distributive lattice $[9,11]$.

Let $R$ be a Riesz space with unit. A base for the topology of the spectrum $\Sigma$ can be given by the opens $\{\sigma \mid \hat{a}(\sigma)>0\}$. In fact, this base can be described explicitly: Let $P$ denote the set of positive elements of $R$. For $a, b$ in $P$ we define $a \preccurlyeq b$ to mean that there exists $n$ such that $a \leqslant n b$. The following proposition is proved in [13] and involves only elementary considerations on Riesz spaces.

Proposition $1 L(R):=(P, \vee, \wedge, 1,0, \preccurlyeq)$ is a distributive lattice. In fact, if we define $D: R \rightarrow L(R)$ by $D(a):=a^{+}$, then $L(R)$ is the free lattice generated by $\{D(a) \mid a \in R\}$ subject to the following relations:

1. $D(a)=0$, if $a \leqslant 0$;

2. $D(1)=1$;

3. $D(a) \wedge D(-a)=0$;

4. $D(a+b) \leqslant D(a) \vee D(b)$;

5. $D(a \vee b)=D(a) \vee D(b)$.

We have $D(a) \leqslant D(b)$ if and only if $a^{+} \preccurlyeq b^{+}$and $D(a)=0$ if and only if $a \leqslant 0$. We write $a \in(p, q):=(a-p) \wedge(q-a)$ and observe that this is an element of $R$.

For $a$ in $R$ we define its norm $\|a\|=\inf \{q \mid a \leqslant q 1\}$.

The corresponding locale ${ }^{2}$ (complete distributive lattice) $\Sigma$ is the one defined by the same generators and relations together with the relation $D(a)=\bigvee_{s>0} D(a-s)$. The generators and relations above may also be read as a propositional geometric theory [27] by reading $\leqslant$ as $\Rightarrow$. A model $m$ of this theory defines a representation $\sigma_{m}$ of the Riesz space by

$$
\sigma_{m}(a):=(\{q \mid m \models D(q-a)\},\{q \mid m \models D(a-q)\}),
$$

where the right hand side is a Dedekind cut in the rationals and hence a real number. Such a $\sigma_{m}$ is a point of the locale $\Sigma$. This motivates the interpretation of $D(a)$ as $\{\sigma \mid$ $\hat{a}(\sigma)>0\}$ : the models which make the proposition $D(a)$ true coincide with the points $\sigma$ such that $\hat{a}(\sigma)>0$. Proving that there are enough such models/points requires the axiom of choice. We avoid this axiom by working with the propositions/opens instead.

Definition 3 A partition of unity is a list $u_{i}$ such that $\sum u_{i}=1$ and $0 \leqslant u_{i} \leqslant 1$. If $u$, $v$ are partitions of unity in an almost f-algebra, then so is $u \cdot v: \sum_{i} \sum_{j} u_{i} v_{j}=1 \cdot \sum_{j} v_{j}$.

Theorem 2 [Localic Stone-Yosida] The map $\hat{\imath}: R \rightarrow \operatorname{Loc}(\Sigma, \mathbb{R})$ defined by the frame map $\hat{a}(p, q):=a \in(p, q)$ is a norm-preserving Riesz morphism. Its image is dense with respect to the uniform topology on $\operatorname{Loc}(\Sigma, \mathbb{R})$.

\footnotetext{
${ }^{1} \mathrm{~A}$ lattice is normal if for all $b_{1}, b_{2}$ such that $b_{1} \vee b_{2}=\top$ there are $c_{1}, c_{2}$ such that $c_{1} \wedge c_{2}=\perp$ and $c_{1} \vee b_{1}=\top$ and $c_{2} \vee b_{2}=\top$. The opens of a normal topological space form a normal lattice.

${ }^{2}$ From this point onwards $\Sigma$ is the spectrum considered as a locale. If we want to treat it as a topological space we write pt $\Sigma$.
} 
Proof The map $\hat{\imath}: R \rightarrow \operatorname{Loc}(\Sigma, \mathbb{R})$ is norm-preserving; see [13]. It remains to prove density. For this consider a natural number $N$ and a continuous $f$ on $\Sigma$ such that $0 \leqslant f \leqslant 1$. We need to find an element $a$ of $R$ such that $\hat{a}$ is close to $f$. The set $\bigcup_{k=0}^{N} f \in((k-1) / N,(k+1) / N)$ covers $\Sigma$. By Proposition 3.1 of [12] there exists a partition of unity $p_{i}$ in the Riesz space such that $\sum p_{i}=1$ and $D\left(p_{i}\right)$ is contained in some open $f \in\left(\left(k_{i}-1\right) / N,\left(k_{i}+1\right) / N\right)$ in $\Sigma$. Concretely,

$$
p_{i} \preccurlyeq\left(f-\left(k_{i}-1\right) / N\right) \wedge\left(\left(k_{i}+1\right) / N-f\right) .
$$

Consequently,

$$
\left|f-\sum k_{i} \widehat{p}_{i}\right|=\left|f \sum \widehat{p}_{i}-\sum k_{i} \widehat{p}_{i}\right|=\left|\sum\left(f-k_{i}\right) \widehat{p}_{i}\right| \leqslant \frac{1}{N} .
$$

The map $\hat{\imath}$ is a Riesz embedding if $R$ is Archimedean.

Corollary 1 There is a norm-preserving Riesz morphism of $R$ into an f-algebra such that the image is dense with respect to the uniform topology.

The axiom of choice implies that compact regular locales have enough points and hence we obtain the more familiar formulation of the theorem by working with the topological space pt $\Sigma$ of the points of the spectrum. However, in practice, only the localic version is needed.

Corollary 2 [Stone-Yosida] The map $\hat{\imath}: R \rightarrow C(\mathrm{pt} \Sigma, \mathbb{R})$ defined by the frame map $\hat{a}(p, q):=a \in(p, q)$ is a norm-preserving Riesz morphism. Its image is dense with respect to the uniform topology on $C(\mathrm{pt} \Sigma, \mathbb{R})$.

\section{The Results}

Definition 4 An almost $f$-algebra is a Riesz space with multiplication such that $a$. $b \geqslant 0$ if $a, b \geqslant 0$, and $a \wedge b=0$ implies $a \cdot b=0$.

If $E$ is a Riesz space, a bilinear map A of $E \times E$ into a vector space $\mathrm{F}$ is called orthosymmetric if

$$
f \wedge g=0 \Rightarrow A(f, g)=0
$$

for all $f, g \in E$.

In the proof of the following theorem we replace the pointwise arguments of Buskes and van Rooij by explicit manupulations with lattices. Theorem 2 indicates that this is possible, however, the following theorem does not formally depend on it.

We use the following theorem $[2,15,19,23]$, which in fact holds for lattice ordered (possibly non-Abelian) groups. Here we specialize it to Riesz spaces. 
Theorem 3 (Refinement) Suppose that $\sum a_{i}=\sum b_{j}$ and $a_{i}, b_{j} \geq 0$. Then there exist $c_{i j} \geq 0$ such that $a_{i}=\sum_{j} c_{i j}, b_{j}=\sum_{i} c_{i j}$ and

$$
\sum_{k=i+1}^{m} c_{k j} \wedge \sum_{k=j+1}^{n} c_{i k}=0, \quad(\text { for all } i<m, j<n) .
$$

Proof First assume that $m=n=2$. Define

$$
c_{11}=a_{1} \wedge b_{1}, \quad c_{12}=a_{1}-c_{11}, \quad c_{21}=b_{1}-c_{11}, \quad c_{22}=a_{2} \wedge b_{2} .
$$

Then $c_{21}=a_{2}-c_{22}$ and $c_{12}=b_{2}-c_{22}$. Moreover, $c_{12} \wedge c_{21}=c_{11}-\left(a_{1} \wedge b_{1}\right)=0$.

We proceed by induction. Suppose that the theorem holds for all $m^{\prime}, n^{\prime}$ with $m^{\prime} \leqslant m, n^{\prime}<n(n \geq 3)$. Write $\sum a_{i}=\left(\sum_{\leqslant n-2} b_{j}\right)+\left(b_{n-1}+b_{n}\right)$. By the induction hypothesis, there exist $c_{i j}, d_{i} \geq 0$ such that $a_{i}=\sum c_{i j}+d_{i}, b_{j}=\sum c_{i j}$ and $b_{n-1}+$ $b_{n}=\sum d_{i}$. We apply the induction hypothesis again, now to the last equation. We obtain $c_{i j} \geq 0$ such that $d_{i}=c_{i, n-1}+c_{i n}, b_{j}=\sum c_{i j}$ and

$$
\sum_{k=i+1}^{m} c_{k j} \wedge\left(\sum_{k=j+1}^{n-2} c_{i k}+d_{i}\right)=0 .
$$

Theorem 4 Let $E$ be a Riesz space with unit and let $F$ be Archimedean. Let $A$ be a orthosymmetric positive bilinear map $E \times E \rightarrow F$. Then for each $\varepsilon$,

$$
|A(f, g)-A(g, f)| \leqslant 8 \varepsilon A(1,1) .
$$

Proof Let $f, g$ be in $E$.

$$
A(f, g)=A\left(f^{+}, g^{+}\right)+A\left(f^{-}, g^{-}\right)-A\left(f^{-}, g^{+}\right)-A\left(f^{+}, g^{-}\right) .
$$

So, it suffices to consider the case $0 \leqslant f, g \leqslant 1$. Let $k$ be a natural number. Define

$$
u_{n}:=\frac{k}{n+1}\left(\left(f-\frac{n}{k}\right)^{+} \wedge \frac{n+1}{k}\right),
$$

whenever $0 \leqslant n<k$. Define $v_{0}:=1-u_{0}$ and $v_{n}:=u_{n}-u_{n+1}$ and $v_{k}:=u_{k}$. The set $\left\{v_{0}, \ldots, v_{k}\right\}$ is a partition of unity - that is, $\sum v_{i}=1$ and $0 \leqslant v_{i} \leqslant 1$. Moreover, $v_{n} \perp v_{m}$, whenever $|n-m|>1$ and such that $\left|f v_{n}-\frac{n}{k} v_{n}\right| \leqslant \frac{1}{k}$. By repeating a similar construction for $g$ we find a partition of unity $v^{\prime}$. Then by the Refinement Theorem we obtain $w_{i j}$, again a partition of unity. We let $n$ range over pairs $i j$ and define $\alpha_{n}, \beta_{n}$ such that $\left|f-\sum_{n} \alpha_{n} w_{n}\right| \leqslant \frac{1}{k}$ and $\left|g-\sum_{n} \beta_{n} w_{n}\right| \leqslant \frac{1}{k}$.

Let $\varepsilon=\frac{1}{k}$. Set $f^{\prime}:=\sum \alpha_{n} w_{n}, g^{\prime}:=\sum \beta_{n} w_{n}$ and $h:=\sum \alpha_{n} \beta_{n} w_{n}$. Then

$$
\begin{aligned}
\left|A(f, g)-A\left(f^{\prime}, g^{\prime}\right)\right| & =\left|A\left(f-f^{\prime}, g\right)+A\left(f^{\prime}, g-g^{\prime}\right)\right| \\
& \leqslant \varepsilon A(1,1)+\varepsilon A(1,1)
\end{aligned}
$$

since $\left|f-f^{\prime}\right|,\left|g-g^{\prime}\right| \leqslant \varepsilon$ and $A$ is positive. Thus, it suffices to show that

$$
\left|A\left(f^{\prime}, g^{\prime}\right)-A(1, h)\right| \leqslant 2 \varepsilon A(1,1) .
$$


Observe that for all $i j, i^{\prime} j^{\prime}$,

if $\left|i-i^{\prime}\right|>1$ or $\left|j-j^{\prime}\right|>1$, then $w_{i j} \perp w_{i^{\prime} j^{\prime}}$, so $A\left(w_{i j}, w_{i^{\prime} j^{\prime}}\right)=0$;

otherwise $\left|\alpha_{n}-\alpha_{m}\right| \leqslant 2 \varepsilon$.

It follows that

$$
\begin{aligned}
\left|A\left(f^{\prime}, g^{\prime}\right)-A(1, h)\right| & =\left|\sum_{n, m} \alpha_{n} \beta_{m} A\left(w_{n}, w_{m}\right)-\sum_{n, m} \alpha_{m} \beta_{m} A\left(w_{n}, w_{m}\right)\right| \\
& \leqslant \sum_{n, m}\left|\alpha_{n}-\alpha_{m}\right|\left|\beta_{m}\right| A\left(w_{n}, w_{m}\right) \\
& \leqslant 2 \varepsilon \sum_{n, m} A\left(w_{n}, w_{m}\right)=2 \varepsilon A(1,1)
\end{aligned}
$$

The last inequality follows from the observation above and $\left|\beta_{m}\right| \leqslant 1$.

Changing the roles of the $\alpha$ s and $\beta$ s we have that $\left|A\left(g^{\prime}, f^{\prime}\right)-A(1, h)\right| \leqslant 2 \varepsilon A(1,1)$. Hence $\left|A\left(f^{\prime}, g^{\prime}\right)-A\left(g^{\prime}, f^{\prime}\right)\right| \leqslant 4 \varepsilon A(1,1)$ and $|A(f, g)-A(g, f)| \leqslant 8 \varepsilon A(1,1)$.

Corollary 3 In the context of the previous theorem, let, moreover, $\bar{E}$ be an f-algebra in which $E$ is dense and let $F^{\prime}$ be the uniform completion of $F$. Then A extends uniquely to a orthosymmetric positive bilinear map from $\bar{E} \times \bar{E}$ to $F^{\prime}$ and $A(f, g)=A(1, f g)$ for all $f, g$ in $E$.

Proof The extension of $A$ exist since $A$ is positive and $E$ is dense in $\bar{E}$. With the notation of the previous theorem,

$$
\left|h-f^{\prime} g^{\prime}\right| \leqslant\left|\sum \alpha_{m} \beta_{m} w_{n} w_{m}-\sum \alpha_{n} \beta_{m} w_{n} w_{m}\right| \leqslant \sum\left|\alpha_{n}-\alpha_{m}\right|\left|\beta_{m}\right| w_{n} w_{m} \leqslant 2 \varepsilon .
$$

Hence $|h-f g|$ is small. Since $F$ is Archimedean, $A(f, g)=A(1, f g)$.

We note that $E$ is dense in the f-algebra $C\left(\Sigma_{E}, \mathbb{R}\right)$.

Corollary 4 Let $E$ and $F$ be Riesz spaces of which $F$ is Archimedean. Let $A$ be an orthosymmetric positive bilinear map $E \times E \rightarrow F$. Then

$$
A(f, g)=A(g, f) \quad(f, g \in E) .
$$

Proof Take $f, g \in E$. Let $E_{0}$ be the Riesz subspace of $E$ generated by $\{f, g\}$. Then $|f|+|g|$ is a unit in $E_{0}$. Without restriction, suppose that $E_{0}$ is $E$. The result now follows from Theorem 4.

We have proved the following result in an explicit way by a straightforward analysis of the proof by Buskes and van Rooij.

Corollary 5 Every Archimedean almost $f$-algebra is commutative. 


\section{Internal Real Numbers}

Buskes and van Rooij use the Stone-Yosida representation theorem combined with Dini's theorem to show that a certain sequence of elements in a Riesz space converges. This can be replaced by applying the following result which does not require the sequence to be decreasing.

Theorem 5 Let $e_{n}$ be a sequence of expressions in the language of Riesz spaces such that $e_{n}$ converges constructively when interpreted in the Riesz space of real numbers. Then $e_{n}$ converges uniformly when interpreted in any Riesz space with strong unit.

Proof The Riesz space can be (densely) embedded into a space $C(\Sigma)$ and hence its elements may be interpreted as global sections of the real number object in the topos $\operatorname{Sh}(\Sigma)$ of sheaves over $\Sigma[18,21]$. Now, if $a_{n}$ converges to 0 in the internal language of $\operatorname{Sh}(\Sigma)$. Then for each $q$ there exists $n$ such that $a_{n} \leqslant q$ internally. This is interpreted as: for each $q$ there exists a (finite) cover $U_{i}$ of $\Sigma$ and $n_{i}$ such that $a_{n_{i}} \leqslant q$ on $U_{i}$. Taking $n=\min n_{i}$ we see that $a_{n} \leqslant q$ on $\Sigma$.

Sheaf theory may seem to be a very complex tool to use for such a simple lemma, however, when applied in concrete cases we obtain natural results. For instance, Buskes and van Rooij apply Dini's theorem and the Stone-Yosida representation theorem to prove that the sequence $[(f \wedge g) h-n f(g \wedge h)]^{+}$converges uniformly. We first prove this for the Riesz space of real numbers. Fix $m$ in $\mathbb{N}$. We may assume that $f, g, h \leqslant 1$. Moreover, either ${ }^{3} f \geqslant \frac{1}{m}$ or $f \leqslant \frac{2}{m}$. We may assume that $f \geqslant \frac{1}{m}$ and similarly that $g, h \geqslant \frac{1}{m}$. Choosing $n=m^{2}$ shows that $(f \wedge g) h \leqslant 1$ and $n f(g \wedge h) \geqslant n \cdot \frac{1}{m} \cdot \frac{1}{m} \geqslant 1$. Hence if $n \geqslant m^{2}$, then $[(f \wedge g) h-n f(g \wedge h)]^{+} \leqslant \frac{2}{m}$ in all cases. The interpretation of this statement in the sheaf model $\operatorname{Sh}(\Sigma)$ defined from the spectrum $\Sigma$ of a Riesz space is: there is a finite cover $U_{i}$ of $\Sigma$ such that $[(f \wedge g) h-n f(g \wedge h)]^{+} \leqslant \frac{2}{m}$ is true on each $U_{i}$. A finite cover gives rise to a partition $u_{i}$ of unity such that $D\left(u_{i}\right) \subset U_{i}$. So, that $u_{i}[(f \wedge g) h-n f(g \wedge h)]^{+} \leqslant \frac{2}{m}$ and hence

$$
[(f \wedge g) h-n f(g \wedge h)]^{+}=\sum u_{i}[(f \wedge g) h-n f(g \wedge h)]^{+} \leqslant \frac{2}{m} .
$$

Takeuti's use of Boolean valued models [26] to obtain non-standard results from familiar theorems has a similar flavor as Theorem 5. Boolean valued models are a special class of sheaf models.

\section{Conclusion}

We have illustrated how the use of locale theory, presented by a normal distributive lattice of basic elements, naturally translates proofs which depend on the axiom of choice to simpler lattice theoretic proofs which avoid the axiom of choice, even in its countable form, and the principle of excluded middle. Buskes and van Rooij had previously proposed different methods to avoid the axiom of choice. An advantage

\footnotetext{
${ }^{3}$ The case distinction $f \geqslant \frac{1}{m}$ or $f \leqslant \frac{1}{m}$ is not constructive/continuous.
} 
of our approach is that it is valid in any topos. It also provides a logical tool to remove the use of representation theorems from Riesz space theory, the importance of avoiding representation theorems was stressed by Zaanen.

Acknowledgements I would like to thank Gerard Buskes, Thierry Coquand and Arnoud van Rooij for comments on an earlier draft of this paper and the referees for their suggestions which improved the presentation of the paper.

Open Access This article is distributed under the terms of the Creative Commons Attribution Noncommercial License which permits any noncommercial use, distribution, and reproduction in any medium, provided the original author(s) and source are credited.

\section{References}

1. Buskes, G., de Pagter, B., van Rooij, A.: Functional calculus on Riesz spaces. Indag. Math. (N.S.) 2(4), 423-436 (1991)

2. Birkhoff, G.: Lattice ordered groups. Ann. Math. 43(2), 298-331 (1942)

3. Banaschewski, B., Mulvey, C.J.: A constructive proof of the Stone-Weierstrass theorem. J. Pure Appl. Algebra, 116(1-3), 25-40 (1997). Special volume on the occasion of the 60th birthday of Professor Peter J. Freyd

4. Banaschewski, B., Mulvey, C.J.: The spectral theory of commutative $C^{*}$-algebras: the constructive Gelfand-Mazur theorem. Quaest. Math. 23(4), 465-488 (2000)

5. Banaschewski, B., Mulvey, C.J.: The spectral theory of commutative $C^{*}$-algebras: the constructive spectrum. Quaest. Math. 23(4), 425-464 (2000)

6. Banaschewski, B., Mulvey, C.J.: A globalisation of the Gelfand duality theorem. Ann. Pure Appl. Logic 137(1-3), 62-103 (2006)

7. Buskes, G., van Rooij, A.: Small Riesz spaces. Math. Proc. Camb. Philos. Soc. 105(3), 523-536 (1989)

8. Buskes, G., van Rooij, A.: Almost $f$-algebras: commutativity and the Cauchy-Schwarz inequality. Positivity 4(3), 227-231 (2000). Positivity and its applications (Ankara, 1998)

9. Cederquist, J., Coquand, T.: Entailment relations and distributive lattices. In: Logic Colloquium '98 (Prague). Lect. Notes Log., vol. 13, pp. 127-139. Assoc. Symbol. Logic, Urbana, IL (2000)

10. Caspers, M., Heunen, C., Landsman, K., Spitters, B.: Intuitionistic quantum logic of an n-level system. Found. Phys. 39(7), 731-759 (2009)

11. Coquand, T.: Compact spaces and distributive lattices. J. Pure Appl. Algebra 184(1), 1-6 (2003)

12. Coquand, T.: About Stone's notion of spectrum. J. Pure Appl. Algebra 197, 141-158 (2005)

13. Coquand, T., Spitters, B.: Formal topology and constructive mathematics: the Gelfand and StoneYosida representation theorems. J. Univers. Comput. Sci. 11(12), 1932-1944 (2005)

14. Coquand, T., Spitters, B.: A constructive proof of Gelfand duality for $\mathrm{C}^{*}$-algebras. Math. Proc. Camb. Philos. Soc. 147(2), 323-337 (2009). doi:10.1017/S0305004109002539

15. Fuchs, L.: Partially Ordered Algebraic Systems. Pergamon Press, Oxford (1963)

16. Heunen, C., Landsman, K., Spitters, B.: A topos for algebraic quantum theory. Commun. Math. Phys. 291(1), 63-110 (2009)

17. Heunen, C., Landsman, N.P., Spitters, B.: Bohrification of Operator Algebras and Quantum Logic (2009)

18. Johnstone, P.T.: Sketches of an Elephant: a Topos Theory Compendium, vol. 2. Clarendon Press (2002)

19. Lorenzen, P.: Abstrakte Begründung der multiplikativen Idealtheorie. Math. Z. 45, 533-553 (1939)

20. Luxemburg, W.A.J., Zaanen, A.C.: Riesz Spaces, vol. I. North-Holland Mathematical Library. North-Holland Publishing Co., Amsterdam (1971)

21. Mac Lane, S., Moerdijk, I.: Sheaves in Geometry and Logic. Universitext. Springer-Verlag, New York (1994) A first introduction to topos theory. Corrected reprint of the 1992 edition

22. Mulvey, C.J.: On the geometry of choice. In: Topological and Algebraic Structures in Fuzzy Sets. Trends Log. Stud. Log. Libr., vol. 20, pp. 309-336. Kluwer Acad. Publ., Dordrecht (2003) 
23. Riesz, F.: Sur quelques notions fondamentales dans la théorie générale des opérations linéaires. Ann. Math. 41(2), 174-206 (1940)

24. Sambin, G.: Intuitionistic formal spaces - a first communication. In: Skordev, D. (ed.) Mathematical Logic and Its Applications, pp. 187-204. Plenum (1987)

25. Stone, M.H.: A general theory of spectra. II. Proc. Nat. Acad. Sci. U. S. A. 27, $83-87$ (1941)

26. Takeuti, G.: Two applications of logic to mathematics. In: Kanô Memorial Lectures, vol. 3. Publications of the Mathematical Society of Japan, No. 13. Iwanami Shoten, Publishers, Tokyo (1978)

27. Vickers, S.: Locales and toposes as spaces. In: Aiello, M., Pratt-Hartmann, I.E., van Benthem, J.F.A.K. (eds.) Handbook of Spatial Logics, chapter 8. Springer (2007)

28. Yosida, K.: On the representation of the vector lattice. Proc. Imp. Acad. Tokyo 18, 339$342(1942)$

29. Zaanen, A.C.: Riesz spaces. II. In: North-Holland Mathematical Library, vol. 30. North-Holland Publishing Co., Amsterdam (1983)

30. Zaanen, A.C.: Introduction to Operator Theory in Riesz Spaces. Springer-Verlag, Berlin (1997) 